\title{
Registry-based stroke research in Taiwan: past and future
}

\author{
Cheng-Yang Hsieh", ${ }^{1,2}$ Darren Philbert Wu ${ }^{3}$, Sheng-Feng Sung ${ }^{3,4}$ \\ 'Department of Neurology, Tainan Sin Lau Hospital, Tainan, Taiwan; ${ }^{2}$ School of Pharmacy, Institute of Clinical Pharmacy and Pharmaceutical \\ Sciences, National Cheng Kung University College of Medicine, Tainan, Taiwan; ${ }^{3}$ Division of Neurology, Department of Internal Medicine, \\ Ditmanson Medical Foundation Chiayi Christian Hospital, Chiayi City, Taiwan; ${ }^{4}$ Department of Nursing, Min-Hwei Junior College of Health Care \\ Management, Tainan, Taiwan
}

Stroke registries are observational databases focusing on the clinical information and outcomes of stroke patients. They play an important role in the cycle of quality improvement. Registry data are collected from real-world experiences of stroke care and are suitable for measuring quality of care. By exposing inadequacies in performance measures of stroke care, research from stroke registries has changed how we manage stroke patients in Taiwan. With the success of various quality improvement campaigns, mortality from stroke and recurrence of stroke have decreased in the past decade. After the implementation of a nationwide stroke registry, researchers have been creatively expanding how they use and collect registry data for research. Through the use of the nationwide stroke registry as a common data model, researchers from many hospitals have built their own stroke registries with extended data elements to meet the needs of research. In collaboration with information technology professionals, stroke registry systems have changed from web-based, manual submission systems to automated fill-in systems in some hospitals. Furthermore, record linkage between stroke registries and administrative claims databases or other existing databases has widened the utility of registry data in research. Using stroke registry data as the reference standard, researchers have validated several algorithms for ascertaining the diagnosis of stroke and its risk factors from claims data, and have also developed a claims-based index to estimate stroke severity. By making better use of registry data, we believe that we will provide better care to patients with stroke.

KEY WORDS: Database, Electronic health records, Medical record linkage, Registries, Research, Stroke

\section{INTRODUCTION}

As has been observed in other developed countries, Taiwan has experienced a significant transition in the epidemiology of stroke [1]. Taking ischemic stroke as an example, the age-standardized incidence of first-ever ischemic stroke has declined from 142.3 to

\section{Correspondence: Sheng-Feng Sung}

Division of Neurology, Department of Internal Medicine, Ditmanson Medical Foundation Chia-Yi Christian Hospital, 539 Zhongxiao Road, East District, Chiayi City 60002, Taiwan

E-mail: richard.sfsung@gmail.com

Received: Jan 14, 2018 / Accepted: Feb 4, 2018 / Published: Feb 4, 2018

This article is available from: http://e-epih.org/

(C) This is an open-access article distributed under the terms of the Creative Commons Attribution License (http://creativecommons.org/licenses/by/4.0/), which permits unrestricted use, distribution, and reproduction in any medium, provided the original work is properly cited.

(C) 2018, Korean Society of Epidemiology
129.5 per 100,000 adults between 2000 and 2011 [2]. The 1-year recurrence rate of stroke among patients with first-ever ischemic stroke decreased even more steeply from 9.6 to $7.8 \%$ during this period [2]. Improved identification and control of vascular risk factors, as well as adequate secondary prevention, may have contributed to the epidemiological trends of stroke in Taiwan $[2,3]$.

Furthermore, the quality of stroke care, as represented by adherence to performance measures for acute ischemic stroke, also improved from 2000 to 2012 [3]. In particular, the rate of thrombolytic therapy for acute ischemic stroke and the rate of door-toneedle time within 60 minutes both significantly increased [4]. Meanwhile, in recent years, stroke has moved from the second to the fourth leading cause of death in Taiwan [5]. Something must have been done right for such progress to have been made, and we speculate that the development of stroke registries and the associated quality improvement initiatives likely played an important role in this progress. In this article, we review what we have learned about stroke registries over the past decade. Specifically, we focus 
on how researchers have used registry data to conduct stroke research, including data sharing with a common data model and linkage between registry data and administrative claims data.

\section{WHAT IS A STROKE REGISTRY?}

A clinical registry is an observational database, usually focusing on a clinical condition, procedure, therapy, or population. A stroke registry can be defined as "an organized system for the collection, storage, retrieval, analysis, and dissemination of information on individual patients who have had a stroke" [6].

An ideal stroke registry is nationwide and enrolls patients from as many participating hospitals as possible in order to increase representativeness and avoid selection bias [7]. For example, the Riks-Stroke register in Sweden, launched in 1994, has covered all hospitals that admit acute stroke patients across the country since 1998 [8]. Appropriate data structure and governance policies are needed to keep a nationwide stroke registry sustainable and operating well. Through the publication and communication of results, a stroke registry should be helpful for improvement of stroke care quality, health policy, and the outcomes of patients [7].

\section{STROKE REGISTRIES AND THE CYCLE OF QUALITY IMPROVEMENT}

Clinical registries are at the center of the cycle of quality improvement in patient care [6,9]. Stroke registries are no exception (Figure 1). For example, the Taiwan Thrombolytic Therapy for Acute Ischemic Stroke (TTT-AIS) register is a therapy-specific stroke registry, aiming at monitoring the safety and effectiveness of recombinant tissue-type plasminogen activator (rt-PA) in Taiwanese stroke patients [10]. Although rt-PA was approved for patients with acute ischemic stroke in Taiwan in 2002, no placebo-controlled,

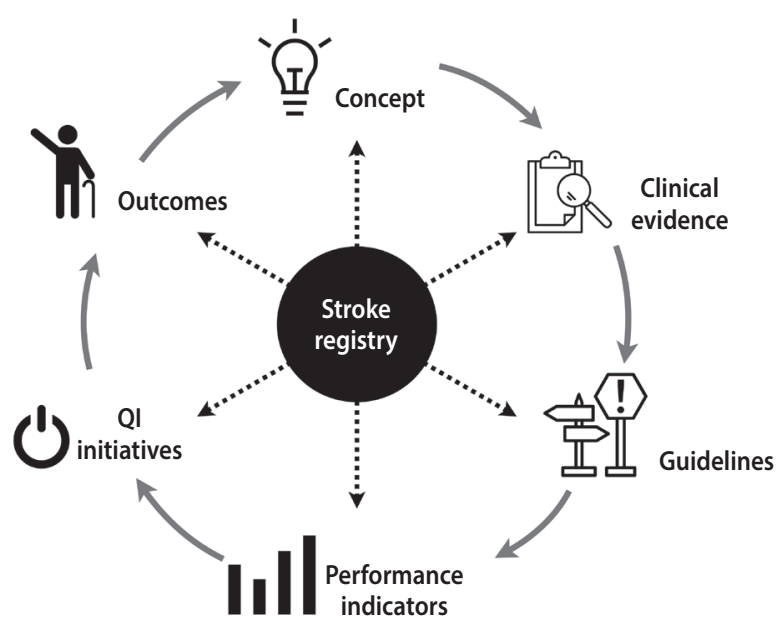

Figure 1. The role of the stroke registry in the cycle of quality improvement. Adapted from Bhatt et al. J Am Coll Cardiol 2015;66:2230-2245 [6]; Califf et al. J Am Coll Cardiol 2002;40:1895-1901 [9]. randomized clinical trials have been conducted on the use of rtPA for acute ischemic stroke in Taiwan or other Asian countries. Concerns remain regarding the safety and effectiveness of rt-PA in Taiwanese patients, for whom the risk of bleeding, including potentially lethal symptomatic intracranial hemorrhage (SICH), may be higher than for Western patients. These concerns lay behind the rationale and concept of the development of the TTTAIS register.

The TTT-AIS register was a centralized and web-based registration system that collected patient data from 23 major hospitals in Taiwan. The first phase of the registry project took place from 2004 through 2008 [10], and the second phase was extended to 2011 [11]. The most salient finding from the TTT-AIS is the interaction between age and rt-PA dose. That is, in patients aged greater than 70 years, a lower dose of rt-PA was associated with less SICH and better functional outcomes [11]. This experience underscores the importance of clinical registries in the cycle of quality improvement. Using registry data, we have turned a concept into clinical evidence, and developed our guidelines accordingly. Additionally, of course, evidence from observational studies should be further examined in randomized controlled trials [12].

\section{TAIWAN STROKE REGISTRY}

Another example illustrating the role of registry-based research in the cycle of quality improvement for stroke care is the Taiwan Stroke Registry (TSR). The TSR is our first large nationwide stroke registry; it was launched in 2006 and is still ongoing. It is also centralized and web-based. The TSR enrolls patients who are admitted within 10 days of stroke onset because of 1 of the 4 major types of stroke (i.e., ischemic stroke, intracerebral hemorrhage, subarachnoid hemorrhage, and transient ischemic attack), and follows them for 6 months afterward. During the early phase of the TSR between 2006 and 2008, a total of 39 hospitals across the country participated in the registration project. Most of them are tertiary referral medical centers and regional hospitals. Data were collected prospectively and entered by TSR-trained study nurses. The TSR employed several quality assurance processes to ensure data quality. About $18 \%$ of the admitted stroke patients in Taiwan were registered during this period [13]. More than 100,000 stroke events have been recorded in the TSR up to 2015 [14]. Nevertheless, the TSR will be more representative of the general population if more hospitals are recruited into the TSR, particularly district hospitals.

The first paper from the TSR was published in Circulation in 2010 [13]. From the title of this paper, we can see the ambitions of the project, which was envisioned to be the "Get With The Guidelines-Stroke" program in Taiwan, the Taiwanese version of the American Heart Association/American Stroke Association Get With The Guidelines-Stroke program. The data presented in that paper documented inadequacies in several performance measures, such as rt-PA for patients with ischemic stroke presenting within 2 hours of onset, anticoagulation for patients with ischemic stroke or transient ischemic attack with atrial fibrillation, and lipid- 
lowering therapy for patients with ischemic stroke or transient ischemic attack with low density lipoprotein-cholesterol $\geq 100 \mathrm{mg} /$ dL [13].

The value of this paper is that it disclosed how we performed on several quality indicators developed from evidence-based guidelines [15]. To address these issues, the Taiwan Joint Commission on Hospital Accreditation undertook an initiative seeking to improve the quality of acute stroke care by adopting the Breakthrough Series (BTS) model [16]. Hospitals interested in improving the quality of acute stroke care were encouraged to participate in the BTS-Stroke activity. A total of 24 major hospitals across the country joined that quality improvement activity during 2010 and 2011. The BTS-Stroke activity consisted of 3 learning sessions and a final summative meeting throughout a 1-year period. Each hospital had a BTS team made up of 3 to 7 core members. Key elements of the BTS activity included topic selection, faculty recruitment, enrollment of participating organizations and teams, learning sessions, action periods, improvement of the model, and outcome measurement and evaluation [16]. With the implementation of this activity, we have observed significant trends of increasing adherence to stroke performance measures and decreasing rates of recurrent stroke and mortality in patients with ischemic stroke in Taiwan $[3,16]$.

\section{RESEARCH FROM THE TAIWAN STROKE REGISTRY}

Although the raw data from the TSR are not open to researchers, site investigators from all participating hospitals can submit their study proposals to the research committee of the TSR. Once study proposals are accepted, staff of the central data laboratory analyze the data and send back the results to investigators for drafting the manuscript. Several research articles from the TSR have been published in prestigious journals, mainly focusing on the predictors of patient-centered outcomes [17-20]. For example, Tang et al. [18] found that low pulse pressure upon admission was associated with poorer 3-month outcomes in patients with acute ischemic stroke. The findings from these studies may provide useful information for the prognostication and management of stroke patients.

However, several limitations of the TSR should be addressed. Because of the scale of the registry, adequate funding is required for its operation and maintenance. Some trade-offs between the comprehensiveness of its data elements and the cost of obtaining quality data are thus inevitable. A few clinically relevant pieces of data are not collected, such as the dose of rt-PA administered, postthrombolytic SICH per various definitions, and the pre-stroke modified Rankin Scale score. Missing data are another common problem for such a registry that tries to keep track of large numbers of patients. Because of shortages of funding and human resources, obtaining follow-up data may be difficult if stroke patients seek medical services in hospitals other than the initial hospital of care and do not come back for follow-up. Furthermore, patients with missing data may have either recovered so well that followup is perceived to be unnecessary or have been placed in a nursing home due to poor functional status, making them unable to return for follow-up. According to a study from the TSR, approximately one-third of the study patients had missing information on 3-month outcomes [18]. Biases may occur if a considerable proportion of data are missing non-randomly.

\section{TAIWAN STROKE REGISTRY AS A COMMON DATA MODEL}

To minimize these problems, enthusiastic researchers from some participating hospitals have tried to combine their data and add more data elements to the existing data structure of the TSR as a workaround. The contribution of the TSR in this regard cannot be overlooked because, by participating in the TSR, many hospitals have built their own stroke registries on top of the common data model set up by the TSR [21]. Despite being based on a much smaller patient pool, several papers drawing on those registries have been published in high-impact journals [22-24]. Lin et al. [22] investigated the readmission risk, causes, and risk factors after discharge in patients with acute stroke. Chen et al. [23] observed that intravenous thrombolysis with standard-dose rt-PA might have a similar profile of safety and effectiveness to that of intravenous thrombolysis with low-dose rt-PA in Taiwanese patients. Sung et al. [24] compared and externally validated several SICH risk-scoring systems, which could help assess the risk of postthrombolysis SICH in patients with acute ischemic stroke. These studies were all intended to answer practical questions about patient care.

\section{STROKE REGISTRIES AND ELECTRONIC MEDICAL RECORDS}

Apart from the TSR, another large stroke registry worth mentioning is the Stroke Registry in Chang Gung Healthcare System (SRICHS). The Chang Gung Medical System (CGMS) is among the largest healthcare groups worldwide, containing 10,000 beds, 8.2 million outpatient visits per year, and more than 3,000 physicians in a network of 7 branch hospitals across the country. It is estimated that a third of the Taiwanese population have sought treatment from the CGMS [25]. More than 4,000 patients with ischemic stroke were admitted to its branch hospitals in a single year [26]. Instead of participating in the TSR, stroke teams in the CGMS developed their own electronic chart-based stroke registry system in 2006, known as the SRICHS [27]. It was innovative at the time it was created because it was the first automated electronic chart-based stroke registry in Taiwan. Stroke patients are automatically enrolled into the SRICHS based on the International Classification of Disease codes at the initial patient encounter. In addition, its data collection mechanism is embedded within the hospital information system. Most of the data elements are automatically filled in with data downloaded from the hospital infor- 
mation system, or manually entered using pull-down menus during the process of medical record writing [27]. Therefore, the SRICHS is more time- and labor-efficient than the TSR. The registration system has been adopted by other medical specialties within the CGMS [28]. Several interesting studies have been published by the SRICHS group [29-31]. The main shortcoming is that the registration system is currently only operable inside the CGMS.

\section{RECORD LINKAGE}

Taiwan's National Health Insurance Research Database (NHIRD), derived from the claims data of Taiwan's National Health Insurance, is widely used in various kinds of clinical research, including studies of risk factors of diseases, outcomes research, healthcare utilization, and patterns of drug prescription [32]. As compared with clinical registries, nationwide administrative claims databases like the NHIRD have several advantages. They generally have a large sample size, contain serial longitudinal data, and are representative of the entire population. They are thus particularly suitable for longitudinal outcome assessment [33].

According to a PubMed search, as of December 2017, more than 200 stroke-related research articles have been published based on data from the NHIRD. However, administrative claims data are collected for insurance reimbursement rather than for the conduct of research. Therefore, data elements in the NHIRD should be properly validated before they can be used in research. Most importantly, the NHIRD lacks information about stroke severity, which is a critical piece of clinical information regarding patients with stroke. Stroke severity varies greatly among patients and largely determines patient outcomes [34]. Consequently, the validity of research findings from claims-based stroke studies might be undermined.

Through record linkage between clinical and claims data using indirect personal identifiers [35], researchers have been trying to find solutions to the problems described above. Cheng et al. [36] and Hsieh et al. [37] separately validated the diagnosis of ischemic stroke in the NHIRD and found that their algorithms could identify cases of ischemic stroke in the NHIRD with a sensitivity of 94.5 to $97.3 \%$ and a positive predictive value of 88.4 to $97.8 \%$. Using hospital records as the reference standard, Cheng et al. [38] established algorithms to verify the mortality status of patients with ischemic stroke in the NHIRD. By linking stroke registry data to the NHIRD, Hanberg et al. [39] evaluated and validated various algorithms to ascertain stroke risk factors in patients with ischemic stroke, transient ischemic attack, or intracerebral hemorrhage. Moreover, using the same record linkage method, Sung et al. [40] developed a claims-based stroke severity index that can be used to estimate the National Institutes of Health Stroke Scale score in patients with ischemic stroke in the NHIRD. This stroke severity index has been separately validated in patients with ischemic stroke [41] and intracerebral hemorrhage [42] and has been applied in several studies of stroke outcomes that have been published in leading journals [43-45].

\section{FUTURE PERSPECTIVES}

While on the one hand, the validated methods for using stroke registry data as the reference standard help corroborate the findings of stroke research based on the NHIRD, record linkage with claims databases may, on the other hand, give a further boost to research based on stroke registry data. Ideally, registry databases with rich and detailed clinical information, once linked with claims databases with long-term outcome data, could offer valuable opportunities for outcomes research (Figure 2). In this way, the strengths of both types of databases can be integrated [33]. For example, despite being applied to a clinical condition other than stroke, record linkage between a clinical registry of patients undergoing 24-hour Holter monitoring and the NHIRD has produced fruitful research results [46,47]. In addition, record linkage will enable us to determine the differences in the baseline characteristics and outcomes between stroke patients admitted to hospitals that do and do not participate in the stroke registry [48], and thus provide an opportunity to examine the generalizability of the results of studies that use registry data. Therefore, linking large-scale stroke registry data to the NHIRD appears to be a promising direction for future registry-based stroke research.

Even though record linkage using multiple indirect personal identifiers is feasible [35], the use of a direct personal identifier (e.g., personal identification numbers) is the gold standard for linking datasets (Figure 2). Record linkage between the NHIRD and other national databases, such as the death registry and birth registry, as well as clinical registry databases using personal identification numbers, has long been made available within the Data Science Center of the Ministry of Health and Welfare located in Taipei. Recently, with advances in network security, researchers have had the opportunity to access and link these national databases via a virtual private network from local branch offices of the Data Science Center across the country. However, many obstacles still lie ahead, including the public's concern for privacy, protests from human rights organizations against the collection and use of health and welfare data, and the amount of red tape necessary to get permission to link data.

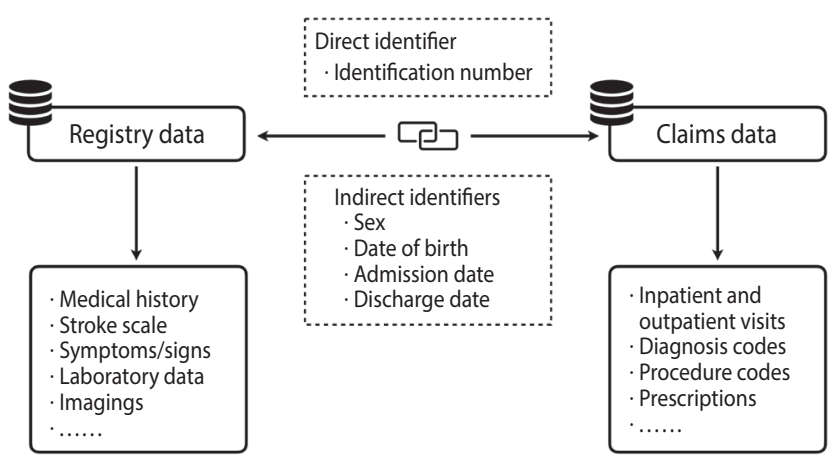

Figure 2. Linkage between registry and claims data using direct or indirect identifiers. 
In this regard, the Registry of Canadian Stroke Network (RCSN) is a useful example. Initially, the registry enrolled only patients who had given written informed consent because of the concern that privacy legislation may mandate informed consent for entering patients into clinical registries. They soon found that, in addition to increasing expenses, obtaining written informed consent led to major selection biases, thus threatening the validity of the registry data [49]. Therefore, under new health privacy legislation in Ontario, Canada, the RCSN was designated as a "prescribed registry," which permitted the RCSN to operate the registry without consent from patients [50]. The RCSN also stopped conducting follow-up interviews with the patients. Instead, patient outcomes are traced by record linkage between the RCSN database and population-based administrative databases using unique encrypted identifiers under the provincial law [50]. Such record linkage may provide a potential remedy for missing data during follow-up.

The successful experience of implementing an electronic chartbased stroke registry system by the SRICHS also inspires us to imagine a broader coverage of data elements in a stroke registry. In the era of big data and the Internet of Things, numerous physiological parameters can be automatically recorded and imported into registry databases. Furthermore, even unstructured medical records can be processed using natural language processing tools to generate useful information [51]. The future development of stroke registries will surely require collaboration between stroke researchers and information technology professionals [52].

Finally, we should seek opportunities to assemble a multinational network of stroke registries in the future. In addition to large sample sizes, multinational stroke registries enable cross-country comparisons and the generalization of study results [14]. For example, similar to that in Taiwan, the healthcare system in South Korea provides coverage to all its citizens. With the aim of describing stroke statistics and the quality of stroke care, a web-based, prospective stroke registry, the Clinical Research Center for Stroke-Fifth Division stroke registry, was launched in 2008 and has been maintained with high quality $[53,54]$. Although registry databases in different countries may be heterogeneous in a variety of aspects, a distributed network approach can be applied [55]. In this approach, databases can be integrated through a common data model without sacrificing the operational independence of individual stroke registries [14], even though we may face challenges in the harmonization of data elements across registries.

\section{CONCLUSION}

Over the past decade, registry-based stroke research and associated quality improvement campaigns have enhanced the quality of care for stroke patients in Taiwan. Record linkage between stroke registries and administrative claims databases has made a significant contribution to claims-based studies by providing methods for ascertaining the diagnosis of stroke and its risk factors, as well as estimating stroke severity, from claims data. In the future, many further possibilities are available if we can make better use of the data we have registered.

\section{CONFLICT OF INTEREST}

The authors have no conflicts of interest to declare for this study.

\section{ORCID}

Cheng-Yang Hsieh: https://orcid.org/0000-0002-8772-4073; Darren Philbert Wu: https://orcid.org/0000-0001-7628-7069; ShengFeng Sung: https://orcid.org/0000-0002-6253-8813

\section{REFERENCES}

1. Institute for Health Metrics and Evaluation. GBD compare; 2015 [cited 2018 Jan 1]. Available from: https://vizhub.healthdata.org/ gbd-compare/.

2. Lee $\mathrm{M}, \mathrm{Wu} \mathrm{YL}$, Ovbiagele B. Trends in incident and recurrent rates of first-ever ischemic stroke in Taiwan between 2000 and 2011. J Stroke 2016;18:60-65.

3. Hsieh CY, Wu DP, Sung SF. Trends in vascular risk factors, stroke performance measures, and outcomes in patients with first-ever ischemic stroke in Taiwan between 2000 and 2012. J Neurol Sci 2017;378:80-84.

4. Cheng TJ, Peng GS, Jhao WS, Lee JT, Wang TH. Nationwide "Hospital Emergent Capability Accreditation by Level-Stroke" improves stroke treatment in Taiwan. J Stroke 2017;19:205-212.

5. Ministry of Health and Welfare. Taiwan's leading causes of death in 2016; 2017 Jun 19 [cited 2018 Jan 1]. Available from https:// www.mohw.gov.tw/dl-33688-712fe414-73fb-4238-bd16-b9c38c949a72.html.

6. Bhatt DL, Drozda JP Jr, Shahian DM, Chan PS, Fonarow GC, Heidenreich PA, et al. ACC/AHA/STS statement on the future of registries and the performance measurement enterprise: a report of the American College of Cardiology/American Heart Association Task Force on Performance Measures and The Society of Thoracic Surgeons. J Am Coll Cardiol 2015;66:2230-2245.

7. Cadilhac DA, Kim J, Lannin NA, Kapral MK, Schwamm LH, Dennis MS, et al. National stroke registries for monitoring and improving the quality of hospital care: a systematic review. Int J Stroke 2016;11:28-40.

8. Asplund K, Hulter Asberg K, Norrving B, Stegmayr B, Terént A, Wester PO, et al. Riks-stroke: a Swedish national quality register for stroke care. Cerebrovasc Dis 2003;15 Suppl 1:5-7.

9. Califf RM, Peterson ED, Gibbons RJ, Garson A Jr, Brindis RG, Beller GA, et al. Integrating quality into the cycle of therapeutic development. J Am Coll Cardiol 2002;40:1895-1901.

10. Chao AC, Hsu HY, Chung CP, Liu CH, Chen CH, Teng MM, et al. Outcomes of thrombolytic therapy for acute ischemic stroke in Chinese patients: the Taiwan Thrombolytic Therapy for Acute Ischemic Stroke (TTT-AIS) study. Stroke 2010;41:885-890.

11. Chao AC, Liu CK, Chen CH, Lin HJ, Liu CH, Jeng JS, et al. Dif- 
ferent doses of recombinant tissue-type plasminogen activator for acute stroke in Chinese patients. Stroke 2014;45:2359-2365.

12. Anderson CS, Robinson T, Lindley RI, Arima H, Lavados PM, Lee TH, et al. Low-dose versus standard-dose intravenous alteplase in acute ischemic stroke. N Engl J Med 2016;374:2313-2323.

13. Hsieh FI, Lien LM, Chen ST, Bai CH, Sun MC, Tseng HP, et al. Get With The Guidelines-Stroke performance indicators: surveillance of stroke care in the Taiwan stroke registry: Get With The Guidelines-Stroke in Taiwan. Circulation 2010;122:1116-1123.

14. Lai EC, Man KK, Chaiyakunapruk N, Cheng CL, Chien HC, Chui CS, et al. Brief Report: databases in the Asia-Pacific region: the potential for a distributed network approach. Epidemiology 2015; 26:815-820.

15. Smith EE, Saver JL, Alexander DN, Furie KL, Hopkins LN, Katzan IL, et al. Clinical performance measures for adults hospitalized with acute ischemic stroke: performance measures for healthcare professionals from the American Heart Association/American Stroke Association. Stroke 2014;45:3472-3498.

16. Hsieh FI, Jeng JS, Chern CM, Lee TH, Tang SC, Tsai LK, et al. Quality improvement in acute ischemic stroke care in Taiwan: the Breakthrough Collaborative in Stroke. PLoS One 2016;11: e0160426.

17. Chen PK, Chiu PY, Tsai IJ, Tseng HP, Chen JR, Yeh SJ, et al. Onset headache predicts good outcome in patients with first-ever ischemic stroke. Stroke 2013;44:1852-1858.

18. Tang SC, Yin JH, Liu CH, Sun MH, Lee JT, Sun Y, et al. Low pulse pressure after acute ischemic stroke is associated with unfavorable outcomes: the Taiwan Stroke Registry. J Am Heart Assoc 2017;6: e005113.

19. Wen CP, Liu CH, Jeng JS, Hsu SP, Chen CH, Lien LM, et al. Prestroke physical activity is associated with fewer post-stroke complications, lower mortality and a better long-term outcome. Eur J Neurol 2017;24:1525-1531.

20. Wang IK, Liu CH, Yen TH, Jeng JS, Sung SF, Huang PH, et al. Renal function is associated with 1-month and 1-year mortality in patients with ischemic stroke. Atherosclerosis 2018:269:288-293.

21. Hsieh CY. Hospital-based stroke registry in Taiwan. Acta Neurol Taiwan 2016;25:73-74.

22. Lin HJ, Chang WL, Tseng MC. Readmission after stroke in a hospital-based registry: risk, etiologies, and risk factors. Neurology 2011;76:438-443.

23. Chen CH, Hsieh CY, Lai TB, Chuang MT, Chen WL, Sun MC. Optimal dose for stroke thrombolysis in Asians: low dose may have similar safety and efficacy as standard dose. J Thromb Haemost 2012;10:1270-1275.

24. Sung SF, Chen SC, Lin HJ, Chen YW, Tseng MC, Chen CH. Comparison of risk-scoring systems in predicting symptomatic intracerebral hemorrhage after intravenous thrombolysis. Stroke 2013; 44:1561-1566

25. Chang Gung Memorial Hospital. Hospital overview [cited 2018 Jan 1]. Available from: http://www.chang-gung.com/en/about. aspx?id $=11 \&$ bid $=1$.

26. Chang KC. Correlation of stroke severity, early recovery, destinations after acute hospitalization and prehospital/posthospital de- lay and length of stay [cited 2018 Jan 1]. Available from: https:// www1.cgmh.org.tw/strokelnk/20100515-ppt/06_990514_KC\%5B1\%5D.pdf.

27. Lee TH, Chang CH, Chang YJ, Chang KC, Chung J; Chang Gung Medical System Stroke Registry Group. Establishment of electronic chart-based stroke registry system in a medical system in Taiwan. J Formos Med Assoc 2011;110:543-547.

28. Chang CH, Lee TH, Chang YJ, Chang KC, Shieh M, Shieh Y. Novel electronic medical record-based stroke registry system; 2014 [cited 2018 Jan 1]. Available from: http://ieeexplore.ieee.org/abstract/document/6775964/.

29. Liu CH, Wei YC, Lin JR, Chang CH, Chang TY, Huang KL, et al. Initial blood pressure is associated with stroke severity and is predictive of admission cost and one-year outcome in different stroke subtypes: a SRICHS registry study. BMC Neurol 2016;16:27.

30. Yeo LLL, Chien SC, Lin JR, Liow CW, Lee JD, Peng TI, et al. Derivation and validation of a scoring system for intravenous tissue plasminogen activator use in Asian patients. J Stroke Cerebrovasc Dis 2017;26:1695-1703.

31. Liu CH, Lin JR, Liou CW, Lee JD, Peng TI, Lee M, et al. Causes of death in different subtypes of ischemic and hemorrhagic stroke. Angiology 2017:3319717738687.

32. Hsing AW, Ioannidis JP. Nationwide population science: lessons from the Taiwan National Health Insurance Research Database. JAMA Intern Med 2015;175:1527-1529.

33. Hammill BG, Hernandez AF, Peterson ED, Fonarow GC, Schulman KA, Curtis LH. Linking inpatient clinical registry data to Medicare claims data using indirect identifiers. Am Heart J 2009;157: 995-1000.

34. Rost NS, Bottle A, Lee JM, Randall M, Middleton S, Shaw L, et al. Stroke severity is a crucial predictor of outcome: an international prospective validation study. J Am Heart Assoc 2016;5:e002433.

35. Setoguchi S, Zhu Y, Jalbert JJ, Williams LA, Chen CY. Validity of deterministic record linkage using multiple indirect personal identifiers: linking a large registry to claims data. Circ Cardiovasc Qual Outcomes 2014;7:475-480.

36. Cheng CL, Kao YH, Lin SJ, Lee CH, Lai ML. Validation of the National Health Insurance Research Database with ischemic stroke cases in Taiwan. Pharmacoepidemiol Drug Saf 2011;20:236-242.

37. Hsieh CY, Chen CH, Li CY, Lai ML. Validating the diagnosis of acute ischemic stroke in a National Health Insurance claims database. J Formos Med Assoc 2015;114:254-259.

38. Cheng CL, Chien HC, Lee CH, Lin SJ, Yang YH. Validity of inhospital mortality data among patients with acute myocardial infarction or stroke in National Health Insurance Research Database in Taiwan. Int J Cardiol 2015;201:96-101.

39. Hanberg JS, Tang WHW, Wilson FP, Coca SG, Ahmad T, Brisco MA, et al. An exploratory analysis of the competing effects of aggressive decongestion and high-dose loop diuretic therapy in the DOSE trial. Int J Cardiol 2017;241:277-282.

40. Sung SF, Hsieh CY, Kao Yang YH, Lin HJ, Chen CH, Chen YW, et al. Developing a stroke severity index based on administrative data was feasible using data mining techniques. J Clin Epidemiol 
2015;68:1292-1300.

41. Sung SF, Hsieh CY, Lin HJ, Chen YW, Chen CH, Kao Yang YH, et al. Validity of a stroke severity index for administrative claims data research: a retrospective cohort study. BMC Health Serv Res 2016;16:509.

42. Hung LC, Sung SF, Hsieh CY, Hu YH, Lin HJ, Chen YW, et al. Validation of a novel claims-based stroke severity index in patients with intracerebral hemorrhage. J Epidemiol 2017;27:24-29.

43. Lu CY, Huang HC, Chang HH, Yang TH, Chang CJ, Chang SW, et al. Acupuncture therapy and incidence of depression after stroke. Stroke 2017;48:1682-1684.

44. Lee M, Saver JL, Wu YL, Tang SC, Lee JD, Rao NM, et al. Utilization of statins beyond the initial period after stroke and 1-year risk of recurrent stroke. J Am Heart Assoc 2017;6:e005658.

45. Chen CM, Yang YH, Chang CH, Chen PC. Effects of transferring to the rehabilitation ward on long-term mortality rate of firsttime stroke survivors: a population-based study. Arch Phys Med Rehabil 2017;98:2399-2407.

46. Lin CY, Chang SL, Lin YJ, Lo LW, Chung FP, Chen YY, et al. Long-term outcome of multiform premature ventricular complexes in structurally normal heart. Int J Cardiol 2015;180:80-85.

47. Yamada S, Lin CY, Chang SL, Chao TF, Lin YJ, Lo LW, et al. Risk of stroke in patients with short-run atrial tachyarrhythmia. Stroke 2017;48:3232-3238.

48. Song S, Fonarow GC, Olson DM, Liang L, Schulte PJ, Hernandez AF, et al. Association of Get With The Guidelines-Stroke program participation and clinical outcomes for Medicare beneficiar- ies with ischemic stroke. Stroke 2016;47:1294-1302.

49. Tu JV, Willison DJ, Silver FL, Fang J, Richards JA, Laupacis A, et al. Impracticability of informed consent in the Registry of the Canadian Stroke Network. N Engl J Med 2004;350:1414-1421.

50. Fang J, Kapral MK, Richards J, Robertson A, Stamplecoski M, Silver FL. The Registry of Canadian Stroke Network: an evolving methodology. Acta Neurol Taiwan 2011;20:77-84.

51. Goryachev S, Sordo M, Zeng QT. A suite of natural language processing tools developed for the I2B2 project. AMIA Annu Symp Proc 2006:931.

52. Paxton EW, Inacio MC, Khatod M, Yue EJ, Namba RS. Kaiser Permanente National Total Joint Replacement Registry: aligning operations with information technology. Clin Orthop Relat Res 2010;468:2646-2663.

53. Kim BJ, Han MK, Park TH, Park SS, Lee KB, Lee BC, et al. Current status of acute stroke management in Korea: a report on a multicenter, comprehensive acute stroke registry. Int J Stroke 2014; 9:514-518.

54. Kim BJ, Park JM, Kang K, Lee SJ, Ko Y, Kim JG, et al. Case characteristics, hyperacute treatment, and outcome information from the clinical research center for stroke-fifth division registry in South Korea. J Stroke 2015;17:38-53.

55. Coloma PM, Schuemie MJ, Trifirò G, Gini R, Herings R, Hippisley-Cox J, et al. Combining electronic healthcare databases in Europe to allow for large-scale drug safety monitoring: the EU-ADR Project. Pharmacoepidemiol Drug Saf 2011;20:1-11. 